\title{
A framework for responsibility
}

\author{
As the world reckons with the news of the first use of genome editing in the human germline, researchers, \\ clinicians, ethicists and policy makers must work across international boundaries to outline a transparent path \\ forward for the responsible translation of this technology in the future.
}

ate last year, while holding a position at the Southern University of Science and Technology of China (Shenzhen), He Jiankui announced at the Second International Summit on Human Gene Editing in Hong Kong that his group had edited the human germline. He deployed CRISPR-Cas9 technology in an in vitro fertilization procedure to target the CCR5 gene for deletion in the hopes of conferring protection against possible HIV infection. The resulting gene-edited embryos gave rise to a successful pregnancy and the birth of twin girls named LuLu and $\mathrm{NaNa}$. In January, Chinese state media confirmed that a second woman had become pregnant with edited embryos from He's experiment. By implanting these edited embryos, He has broken a number of recommendations that are internationally agreed upon, including those issued by the 2015 International Summit on Human Gene Editing, regarding how or whether precision genome engineering of the human germline might occur. As this issue went to press, media were reporting that He had been fired from his university post, and an investigation had concluded that he had evaded supervision in the course of this experiment. While the world reckons with this announcement and awaits a detailed review of the work in public forums, we must also acknowledge that human germline genome editing will likely occur again, and so the need for a well-defined approval and regulatory path for the transparent, responsible translation of this promising technology is urgently needed.

In the wake of He's announcement, some members of biomedical communities centered on genome editing have voiced support for a moratorium on the implantation of genome-edited human embryos until a set of safety requirements is established alongside an analysis of risks, benefits and ethical issues. Funding agencies, such as the US National Institutes of Health, have reiterated that they do not support human germline editing; also, existing laws in many countries, such as the United States, Canada and Germany, provide a legal moratorium that already bans such work. In some countries, including China, enforceable bans have not been put in place.

Medical societies like the American Society of Human Genetics and the International Society for Stem Cell Research have reaffirmed their previously stated position that germline genome editing is not ready for clinical use in humans, especially in the absence of more rigorously monitored laboratory testing of these technologies in human embryos in vitro. The organizers of last year's International Summit on Human Gene Editing also issued a strong rebuke of He's work in their summary statement, echoing their original assessment that any use of germline editing in humans would be irresponsible given that our scientific understanding of these techniques and the requirements for clinical implementation are too uncertain at present and pose too many risks. However, rather than recommend a total moratorium, their statement also provided a brief section proposing a set of requirements that need to be addressed in order to enable a translational pathway. These include the establishment of "standards for preclinical evidence and accuracy of gene modification, assessment of competency for practitioners of clinical trials, enforceable standards of professional behavior, and strong partnerships with patients and patient advocacy groups."

A broad moratorium on germline editing may ultimately be counterproductive and did not prevent He's group from completing their work in secrecy. Given this reality, some scientists favor establishing a global framework within which future considerations of human germline genome editing could be registered and undergo clinical and ethical evaluation. Indeed, this kind of close ethical review has already been proposed in the United Kingdom by the Nuffield Council on Bioethics, and the Chinese Ministry of education has recently announced a gene-editing self-inspection notice that may help catalog any ongoing or future uses of gene-editing technologies on human embryos.
In looking forward, there is a clear and urgent need for the specific elements of this proposed translational pathway to be put in place. There is wide agreement that He's editing approach does not address a compelling unmet clinical need. Although He has said that the father of LuLu and $\mathrm{NaNa}$ was HIV positive, the chances of paternal transmission of the virus are essentially nonexistent under established conditions for in vitro fertilization. To protect against this kind of ill-advised application of the technology, scientific regulators worldwide need to outline defined criteria constituting the types of unmet clinical needs (i.e., diseases and disorders) that are most suitable for potential applications of human genome editing. Examples of this include dominantly inherited genetic disorders with no available disease-modifying therapies, such as certain forms of congenital hearing or vision loss, and these standards need to be communicated clearly to international communities. In addition to defining which diseases are suitable for targeting, regulators also need to establish thresholds of preclinical evidence to ensure that a given locus constitutes a genetic target with the best chance to modify disease risk.

As with any experimental clinical trial, prescribed endpoints to assess target engagement as well as efficacy of the intervention must be well defined from the outset. Investigators and internal review boards overseeing trials involving germline editing need to pay close attention to trial design and patient recruitment methods and ensure that long-term monitoring of the children resulting from these procedures is occurring. Establishing specific international mechanisms, such as a global registry of human germline genome editing, will help to ensure that this promising technology is used in the most ethically responsible and transparent fashion to help address pressing medical needs.

Published online: 6 February 2019 https://doi.org/10.1038/s41591-019-0352-3 